\title{
ON A FAMILY OF REPRESENTATIONS OF RESIDUALLY FINITE GROUPS
}

\author{
V. MANUILOV
}

\begin{abstract}
For a residually finite group $G$, its normal subgroups $G \supset G_{1} \supset G_{2} \ldots$ with $\cap_{n \in \mathbb{N}} G_{n}=\{e\}$ and for a growth function $\gamma$ we construct a unitary representation $\pi_{\gamma}$ of $G$. For the minimal growth, $\pi_{\gamma}$ is weakly equivalent to the regular representation, and for the maximal growth it is weakly equivalent to the direct sum of the quasiregular representations on the quotients $G / G_{n}$. In the case of intermediate growth we show two examples of different behaviour of $\pi_{\gamma}$.
\end{abstract}

\section{INTRODUCTION}

Let $G$ be a residually finite group with normal subgroups $G \supset G_{1} \supset G_{2} \cdots$ such that $\cap_{n \in \mathbb{N}} G_{n}=\{e\}$. Let $X_{n}=G / G_{n}$ be the quotient finite group, with the natural action of $G$ denoted by $(g, x) \rightarrow g x, g \in G, x \in X_{n}$. Let $\nu_{n}=\left|X_{n}\right|$, where $|A|$ denotes the number of elements in the set $A$. A sequence $\left(\gamma_{n}\right)_{n \in \mathbb{N}}$ of integers such that $1 \leq \gamma_{n} \leq \nu_{n}$ for any $n \in \mathbb{N}$ will be referred to as a growth function.

It is well known that the regular representation $\lambda_{G}$ of $G$ is weakly contained in the direct sum of quasi-regular representations $\lambda_{n}$, which factorize through the quotients $G / G_{n}$, i.e. $\left\|\lambda_{G}(a)\right\| \leq \sup _{n \in \mathbb{N}}\left\|\lambda_{n}(a)\right\|$ for any $a \in \mathbb{C}[G]$. In view of abundance of exotic group norms [2, 6], we were interested to find intermediate norms between the norms $\left\|\lambda_{G}(\cdot)\right\|$ and $\sup _{n \in \mathbb{N}}\left\|\lambda_{n}(\cdot)\right\|$ (in the case of non-amenable $G$ - otherwise these two norms coincide). We had not succeeded, but we have constructed a family $\pi_{\gamma}, \gamma \in Z$, of representations on Hilbert spaces $H_{\gamma}$, where $Z$ is a partially ordered set of growth functions, such that $\pi_{\gamma}$ is weakly equivalent to $\lambda$ for the minimal growth function $\left(\gamma_{n}=1\right.$ for any $\left.n \in \mathbb{N}\right)$, and to $\oplus_{n \in \mathbb{N}} \lambda_{n}$ for the maximal growth function $\left(\gamma_{n}=\nu_{n}\right.$ for any $\left.n \in \mathbb{N}\right)$. The most interesting case is that of intermediate growth. We prove an estimate on the norm of $\pi_{\gamma}$ when the growth function $\gamma$ is sufficiently slow. We also determine, in two cases, whether the trivial representation is weakly contained in $\pi_{\gamma}$. For a property $(\tau)$ group $G$ the answer is negative for all growth functions that are slower than the maximal one, but for a certain choice of finite index subgroups of a free group the answer is positive for some $\gamma$ of an intermediate growth. We were not able to check if the norms $\left\|\pi_{\gamma}(\cdot)\right\|$ are all different. We hope they are; if not, then there should be a boundary growth function such that slower growth functions give the regular norm, and faster ones give the norm $\sup _{n \in \mathbb{N}}\left\|\lambda_{n}(\cdot)\right\|$.

\section{Construction of a family of Representations}

In this section we modify the Calkin's construction [3] to obtain representations of certain quotient $C^{*}$-algebras.

Let $l^{2}\left(X_{n}\right), n \in \mathbb{N}$, be the finitedimensional Hilbert spaces with respect to the atomic measure $\mu$ given by $\mu(x)=1$ for any $x \in X_{n}$. Set $\sigma=\oplus_{n \in \mathbb{N}} \lambda_{n}$, and let $\mathcal{A}=C_{\sigma}^{*}(G)$ be the $C^{*}$-algebra generated by all $\sigma(g), g \in G$. Note that $\sigma$ is a representation of $G$ on the Hilbert space $\oplus_{n \in \mathbb{N}} l^{2}\left(X_{n}\right)$, and there is a canonical inclusion $\mathcal{A} \subset \prod_{n \in \mathbb{N}} \mathbb{B}\left(l^{2}\left(X_{n}\right)\right) \subset$ $\mathbb{B}\left(\oplus_{n \in \mathbb{N}} l^{2}\left(X_{n}\right)\right)$. 
Fix some non-principal ultrafilter $\mathcal{U}$ on $\mathbb{N}$ and let $\tau_{n}$ be the trace on $\mathbb{B}\left(l^{2}\left(X_{n}\right)\right)$ normalized by $\tau_{n}(\mathrm{id})=1$. Let $J \subset \prod_{n \in \mathbb{N}} \mathbb{B}\left(l^{2}\left(X_{n}\right)\right)$ be the ideal consisting of all sequences $\left(m_{n}\right)_{n \in \mathbb{N}}, m_{n} \in \mathbb{B}\left(l^{2}\left(X_{n}\right)\right)$, such that $\lim _{\mathcal{U}} \tau_{n}\left(m_{n}^{*} m_{n}\right)=0$. Then $I=J \cap \mathcal{A}$ is an ideal in $\mathcal{A}$.

Following Calkin, set

$$
\widetilde{H}=\left\{\xi=\left(\xi_{n}\right)_{n \in \mathbb{N}}: \xi_{n} \in l^{2}\left(X_{n}\right), \sup _{n \in \mathbb{N}}\left\|\xi_{n}\right\|<\infty\right\}
$$

and define a sesquilinear form on $\widetilde{H}$ by $\langle\xi, \eta\rangle=\lim _{\mathcal{U}}\left\langle\xi_{n}, \eta_{n}\right\rangle$, where $\xi_{n}, \eta_{n} \in l^{2}\left(X_{n}\right)$, $\xi=\left(\xi_{n}\right)_{n \in \mathbb{N}}, \eta=\left(\eta_{n}\right)_{n \in \mathbb{N}} \in \widetilde{H}$.

Set $\widetilde{H}_{0}=\left\{\xi \in \widetilde{H}: \lim _{\mathcal{U}}\langle\xi, \xi\rangle=0\right\} \subset \widetilde{H}$. When passing to the quotient linear space $\widetilde{H} / \widetilde{H}_{0}$, the sesquilinear form becomes positive-definite, hence defines an inner product. Completion of $\widetilde{H} / \widetilde{H}_{0}$ with respect to the corresponding norm is a (non-separable) Hilbert space $\widehat{H}$.

The sequence $\lambda_{n}, n \in \mathbb{N}$, defines a unitary representation $\widehat{\lambda}$ of the $C^{*}$-algebra $A$ on $\widetilde{H}$ by $\widehat{\lambda}(g)\left(\xi_{n}\right)=\left(\lambda_{n}(g) \xi_{n}\right)$, and it is well-known due to Calkin [3] that this representation annulates the ideal $I_{0}$ in $A$ consisting of sequences $\left(m_{n}\right)_{n \in \mathbb{N}}$ that vanish at infinity, i.e. $\lim _{n \rightarrow \infty}\left\|m_{n}\right\|=0$.

This ideal is smaller than $I$. One of our aims is to find a subspace $H_{1} \subset \widehat{H}$ invariant under $\widehat{\lambda}$ such that $\left.\widehat{\lambda}\right|_{H_{1}}$ would annulate $I$.

Recall that $\nu_{n}=\left|X_{n}\right|$, and, by the definition, $\frac{\nu_{n+1}}{\nu_{n}} \geq 2$, so $\lim _{n \rightarrow \infty} \nu_{n}=\infty$. Let $Z$ denote the set of all non-decreasing integer-valued sequences $\gamma=\left(\gamma_{n}\right)_{n \in \mathbb{N}}$ such that $1 \leq \gamma_{n} \leq \nu_{n}$ for any $n \in \mathbb{N}$. For $\gamma, \gamma^{\prime} \in Z$, we write $\gamma \leq \gamma^{\prime}$ if there is $\varepsilon>0$ such that $\varepsilon \gamma_{n} \leq \gamma_{n}^{\prime}$ for any $n \in \mathbb{N}$, and $\gamma \sim \gamma^{\prime}$ if $\gamma \leq \gamma^{\prime}$ and $\gamma^{\prime} \leq \gamma$. The set $Z$ contains the minimal element $\iota$ such that $\iota_{n}=1$ for any $n \in \mathbb{N}$, and the maximal element $\nu\left(\nu_{n}=\left|X_{n}\right|\right.$ for any $n \in \mathbb{N}$ ). We write $\gamma \prec \gamma^{\prime}$ if $\lim _{\mathcal{U}} \frac{\gamma_{n}}{\gamma_{n}^{\prime}}=0$.

Fix $\gamma \in Z$. Let $\widetilde{H}_{\gamma}^{(k)} \subset \widetilde{H}$ be the subset of all sequences $\left(\xi_{n}\right)$ such that $\left|\operatorname{supp} \xi_{n}\right| \leq k \gamma_{n}$ for each $n$. This is not a linear subspace, but $\widetilde{H}_{\gamma}=\cup_{k=1}^{\infty} \widetilde{H}_{\gamma}^{(k)}$ is. Indeed, if $\left(\xi_{n}\right) \in \widetilde{H}_{\gamma}^{(k)}$, $\left(\xi_{n}^{\prime}\right) \in \widetilde{H}_{\gamma}^{\left(k^{\prime}\right)}$ then $\left(\xi_{n}+\xi_{n}^{\prime}\right) \in \widetilde{H}_{\gamma}^{\left(k+k^{\prime}\right)}$

Let $H_{\gamma}$ denote the closure of $\left(\widetilde{H}_{\gamma}^{(k)}+\widetilde{H}_{0}\right) / \widetilde{H}_{0} \subset \widehat{H}$. Note that if $\gamma, \gamma^{\prime} \in Z, \gamma \leq \gamma^{\prime}$, then $H_{\gamma} \subset H_{\gamma^{\prime}}$, hence $H_{\gamma}=H_{\gamma^{\prime}}$ if $\gamma \sim \gamma^{\prime}$. If $\gamma=\nu$ then $H_{\nu}=\widehat{H}$.

\section{Some Properties of the Hilbert spaces $H_{\gamma}$}

Lemma 2.1. Let $\gamma \prec \gamma^{\prime}$. Then $H_{\gamma} \neq H_{\gamma^{\prime}}$.

Proof. Assume the contrary, and let $E_{n}^{\prime} \subset X_{n},\left|E_{n}^{\prime}\right|=\gamma_{n}^{\prime}$. Take $\xi=\left(\xi_{n}\right) \in \widetilde{H}$, with $\xi_{n}=\frac{1}{\sqrt{\gamma_{n}^{\prime}}} \chi_{E_{n}^{\prime}}$, where $\chi_{E}$ denotes the characteristic function of the set $E$. Then, for any $\varepsilon \in(0,1)$ there exists $k \in \mathbb{N}$ and $\eta \in \widetilde{H}_{\gamma}^{(k)}$ such that $\|\xi-\eta\|<\varepsilon$. Let $E_{n}=\operatorname{supp} \eta_{n}$, $\left|E_{n}\right| \leq k \gamma_{n}$. Then

$$
\begin{aligned}
\frac{\gamma_{n}^{\prime}-k \gamma_{n}}{\gamma_{n}^{\prime}} & \leq \frac{\left|E_{n}^{\prime} \backslash E_{n}\right|}{\gamma_{n}^{\prime}}=\sum_{x \in E_{n}^{\prime} \backslash E_{n}}\left|\xi_{n}(x)\right|^{2} \\
& \leq \sum_{x \in E_{n}^{\prime} \backslash E_{n}}\left|\xi_{n}(x)\right|^{2}+\sum_{x \in E_{n}}\left|\xi_{n}(x)-\eta_{n}(x)\right|^{2}=\left\|\xi_{n}-\eta_{n}\right\|^{2}<\varepsilon^{2},
\end{aligned}
$$

hence $k \frac{\gamma_{n}}{\gamma_{n}^{\prime}}>1-\varepsilon^{2}$. Then $0=k \lim _{\mathcal{U}} \frac{\gamma_{n}}{\gamma_{n}^{\prime}} \geq 1-\varepsilon^{2}$, a contradiction with $\gamma \prec \gamma^{\prime}$. 
Remark 2.2. Note that the same argument shows that $H_{\gamma}$ is strictly greater than the closure of the union of all $H_{\gamma^{\prime}}$ over all $\gamma^{\prime}$ such that $\gamma^{\prime} \prec \gamma$.

Lemma 2.3. Let $\eta=\left(\eta_{n}\right)_{n \in \mathbb{N}} \in \widetilde{H}$. If $\eta \in \widetilde{H}_{1}$ and $\lim _{\mathcal{U}}\left\|\eta_{n}\right\|_{\infty}=0$ then $\eta \in \widetilde{H}_{0}$.

Proof. It suffices to prove Lemma in the case when $\eta \in \widetilde{H}_{1}^{(k)}$ for some $k \in \mathbb{N}$. This means that $\left|\operatorname{supp} \eta_{n}\right| \leq k$ for any $n \in \mathbb{N}$. Then $\left\|\eta_{n}\right\|^{2} \leq k\left\|\eta_{n}\right\|_{\infty}^{2}$.

Remark 2.2 shows that the family of Hilbert spaces is not lower semicontinuous. Now let us show that it is upper semicontinuous.

Proposition 2.4. For any $\gamma^{0} \in Z$ one has $H_{\gamma^{0}}=\cap_{\gamma \succ \gamma^{0}} H_{\gamma}$.

Proof. Let $\xi \in \cap_{\gamma \succ \gamma^{0}} H_{\gamma}$ be represented by a sequence $\left(\xi_{n}\right)_{n \in \mathbb{N}}$. For any $\varepsilon>0$ and for any $n \in \mathbb{N}$, consider all $\eta_{n} \in l^{2}\left(X_{n}\right)$ such that $\left\|\xi_{n}-\eta_{n}\right\|<\varepsilon$. Among all these $\eta_{n}$ one can find $\eta_{n}^{\prime}$ with minimal possible $\left|\operatorname{supp} \eta_{n}\right|$. Set $\alpha_{n}^{\varepsilon}=\left|\operatorname{supp} \eta_{n}^{\prime}\right|$.

Since $\xi \in \cap_{\gamma \succ \gamma^{0}} H_{\gamma}$, for any $\varepsilon>0$ and for any $\gamma \succ \gamma^{0}$ there exists $k \in \mathbb{N}$ and $\zeta \in \widetilde{H}_{\gamma}^{(k)}$ such that $\|\xi-\zeta\|<\varepsilon$. In particular, $\left|\operatorname{supp} \zeta_{n}\right| \leq k \gamma_{n}$ for all $n \in \mathbb{N}$.

It follows from $\|\xi-\zeta\|<\varepsilon$ that there exists $\mathbb{A} \in \mathcal{U}$ sich that $\left\|\xi_{n}-\zeta_{n}\right\|<\varepsilon$ for any $n \in \mathbb{A}$. By assumption, $\alpha_{n}^{\varepsilon} \leq\left|\operatorname{supp} \zeta_{n}\right| \leq k \gamma_{n}$ for any $n \in \mathbb{A}$. Thus, for any $\varepsilon>0$ and any $\gamma \succ \gamma^{0}$ there exists $k \in \mathbb{N}$ and $\mathbb{A} \in \mathcal{U}$ such that $\alpha_{n}^{\varepsilon} \leq k \gamma_{n}$ for any $n \in \mathbb{A}$.

Suppose that $\lim _{\mathcal{U}} \frac{\alpha_{n}^{\varepsilon}}{\gamma_{n}^{0}}=\infty$ for some $\varepsilon>0$. Then set $\gamma_{n}^{\prime}=\sqrt{\alpha_{n}^{\varepsilon} \gamma_{n}^{0}}$. By assumption, $\lim _{\mathcal{U}} \frac{\gamma_{n}^{\prime}}{\gamma_{n}^{0}}=\lim _{\mathcal{U}} \sqrt{\frac{\alpha_{n}^{\varepsilon}}{\gamma_{n}^{0}}}=\infty$, hence $\gamma^{\prime} \succ \gamma^{0}$. As we have already shown, there exists $k \in \mathbb{N}$ and $\mathbb{A} \in \mathcal{U}$ such that $\alpha_{n}^{\varepsilon} \leq k \gamma_{n}^{\prime}=k \sqrt{\alpha_{n}^{\varepsilon} \gamma_{n}^{0}}$ for any $n \in \mathbb{A}$, hence $\alpha_{n}^{\varepsilon} \leq k^{2} \gamma_{n}^{0}$. This contradicts our assumption.

Thus, for any $\varepsilon>0$ we have $\lim _{\mathcal{U}} \frac{\alpha_{n}^{\varepsilon}}{\gamma_{n}^{0}}<\infty$, i.e. for any $\varepsilon>0$ there exists $C \in \mathbb{R}$

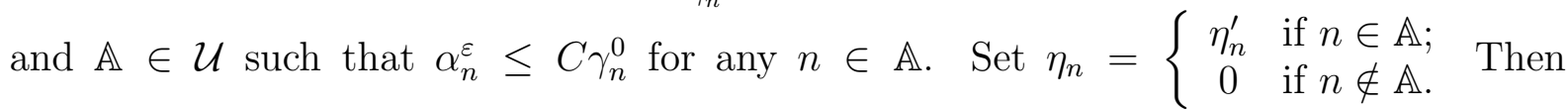
$\eta=\left(\eta_{n}\right)_{n \in \mathbb{N}} \in H_{\gamma^{0}}$, and $\|\xi-\eta\|=\lim _{\mathcal{U}}\left\|\xi_{n}-\eta_{n}\right\|<\varepsilon$. As $\varepsilon$ is arbitrary, this implies that $\xi \in H_{\gamma^{0}}$ and we are done.

Lemma 2.5. The subspaces $H_{\gamma}$ are invariant under $\widehat{\lambda}$.

Proof. Obvious: translation by $g \in G$, of functions in $l^{2}\left(X_{n}\right)$ does not change the size of their supports.

Thus, we can restrict the representation $\widehat{\lambda}$ to $H_{\gamma}$ for any $\gamma \in Z$. Denote this restriction by $\pi_{\gamma}=\left.\widehat{\lambda}\right|_{H_{\gamma}}$.

Note that if $\gamma \leq \gamma^{\prime}$ then $H_{\gamma} \subset H_{\gamma^{\prime}}$, hence the representation $\pi_{\gamma^{\prime}}$ contains the representation $\pi_{\gamma}$.

\section{Case of maximal growth}

Theorem 3.1. The representations $\pi_{\nu}$ and $\oplus_{n \in \mathbb{N}} \lambda_{n}$ are weakly equivalent.

Proof. When $\gamma=\nu$ is maximal, there is no restrictions on the size of supports of vectors in $\widetilde{H}$, so $\pi_{\nu}=\widehat{\lambda}$. The classical result of J. W. Calkin [3] states that the kernel of the representation $\hat{\lambda}$ of $\mathcal{A}$ on $\widehat{H}$ is $\mathcal{A} \cap \mathbb{K}\left(\oplus_{n \in \mathbb{N}} l^{2}\left(X_{n}\right)\right.$ ) (recall that $\mathcal{A}$ is generated by $\oplus_{n \in \mathbb{N}} \lambda_{n}(g)$, $g \in G)$. As $\lambda_{n}$ is a subrepresentation in any $\lambda_{m}$ with $m>n$, each $\lambda_{n}$ repeats infinitely in $\sigma=\oplus_{n \in \mathbb{N}} \lambda_{n}$, hence $\|\widehat{\lambda}(\cdot)\|=\sup _{n \in \mathbb{N}}\left\|\lambda_{n}(\cdot)\right\|$. 


\section{CASE OF VERY SLOW GROWTH}

Theorem 4.1. There exists a growth function $\gamma$ with $\lim _{n \rightarrow \infty} \gamma_{n}=\infty$ such that $\pi_{\gamma^{\prime}}$ is weakly equivalent to the regular representation $\lambda$ of $G$ for any $\gamma^{\prime} \leq \gamma$. In particular, $\pi_{\iota}$ is weakly equivalent to $\lambda$.

Proof. It suffices to prove the theorem in the case when $G$ is finitely generated. Let $q_{n}: G \rightarrow G / G_{n}=X_{n}$ denote the quotient maps, and let $l$ (resp. $l_{n}$ ) be the word length function on $G$ (resp. on $X_{n}$ ) with respect to a fixed set $S \subset G$ (resp. $q_{n}(S) \subset X_{n}$ ) of generators, and let $d(g, h)=l\left(g^{-1} h\left(\operatorname{resp} . d_{n}(x, y)=l_{n}\left(x^{-1} y\right)\right)\right.$ determine a left invariant metric on $G$ (resp. on $X_{n}$ ). Let $B_{r} \subset G$ denote the ball of radius $r$. Let $a \in \mathbb{C}[G]$, supp $a \subset B_{r}$. For any $\gamma \in Z$ and for any $\varepsilon>0$ there exists $\xi \in \widetilde{H}_{\gamma}^{(k)}$ such that $\left\|\xi_{n}\right\|=1$ for any $n \in \mathbb{N}$ and

$$
\left\|\pi_{\gamma}(a)\right\|^{2}<\left\|\pi_{\gamma}(a) \xi\right\|^{2}+\varepsilon .
$$

Let $\operatorname{supp} \xi_{n}=A \subset X_{n}$. By assumption, $|A| \leq k \gamma_{n}$ for any $n \in \mathbb{N}$. Our aim is to show that although $A$ may be scattered on $X_{n}$, one can replace $\xi_{n}$ by another functions $\zeta_{n}$ such that $\left\|\pi_{\gamma}(a)\right\|^{2}<\left\|\pi_{\gamma}(a) \zeta\right\|^{2}+\varepsilon,\left\|\zeta_{n}\right\|=1, n \in \mathbb{N}$, and such that $\operatorname{supp} \zeta_{n}$ lies in a ball of a controlled radius.

Decompose the set $A$ into a disjoint union of its subsets $A=A_{1} \sqcup \cdots \sqcup A_{k}$ such that

(A1) For any $x \in A_{i}, i=1, \ldots, k$, there exists $y \in A_{i}$ such that $\rho(x, y)<3 r$;

(A2) If $x \in A_{i}, y \in A_{j}, i \neq j$, then $\rho(x, y) \geq 3 r$.

Set $\left.\xi_{n}\right|_{A_{i}}=\eta_{i}$. Then $\left\|\eta_{1}\right\|^{2}+\cdots+\left\|\eta_{k}\right\|^{2}=\left\|\xi_{n}\right\|^{2}=1$ and, as $\operatorname{supp} x^{*} x \subset B_{2 r}$, so by (A2), one has $\left\langle\lambda_{n}\left(a^{*} a\right) \eta_{i}, \eta_{j}\right\rangle=0$ when $i \neq j$, hence

$$
\left\|\lambda_{n}(a) \xi_{n}\right\|^{2}=\sum_{i=1}^{k}\left\langle\lambda_{n}\left(a^{*} a\right) \eta_{i}, \eta_{i}\right\rangle
$$

Suppose that for any $i=1, \ldots, k$ one has

$$
\left\|\lambda_{n}\left(a^{*} a\right) \eta_{i}, \eta_{i}\right\| \leq\left(\left\|\lambda_{n}\left(a^{*} a\right)\right\|-\varepsilon\right)\left\|\eta_{i}\right\|^{2} .
$$

Then, summing (4.2) up, we get

$$
\left\|\lambda_{n}(a) \xi_{n}\right\|^{2}=\sum_{i=1}^{k}\left\|\lambda_{n}\left(a^{*} a\right) \eta_{i}, \eta_{i}\right\| \leq\left(\left\|\lambda_{n}\left(a^{*} a\right)\right\|-\varepsilon\right) \sum_{i=1}^{k}\left\|\eta_{i}\right\|^{2}=\left\|\lambda_{n}\left(a^{*} a\right)\right\|-\varepsilon .
$$

Passing to the limit over $\mathcal{U}$, we get

$$
\left\|\pi_{\gamma}(a) \xi\right\|^{2} \leq\left\|\pi_{\gamma}(a)\right\|^{2}-\varepsilon,
$$

which contradicts (4.1). Thus, for any $n \in \mathbb{N}$, there exists $i$ such that

$$
\left\|\lambda_{n}\left(a^{*} a\right) \eta_{i}, \eta_{i}\right\| \leq\left(\left\|\lambda_{n}\left(a^{*} a\right)\right\|-\varepsilon\right)\left\|\eta_{i}\right\|^{2} .
$$

Note that $\left|\operatorname{supp} \eta_{i}\right|=\left|A_{i}\right| \leq|A| \leq k \gamma_{n}$, hence, by (A1), there exists a ball $\widetilde{B}_{r^{\prime}}\left(z_{n}\right)$ of radius $r^{\prime}=k \gamma_{n} \cdot 3 r$ centered at some $z_{n} \in X_{n}$, such that supp $\eta_{i} \subset \widetilde{B}_{r^{\prime}}\left(z_{n}\right)$ (we use tilde to distinguish balls in $X_{n}$ from balls in $G$ ).

Set $\zeta_{n}=\frac{\eta_{i}}{\left\|\eta_{i}\right\|}$. Then $\operatorname{supp} \zeta_{n} \subset \widetilde{B}_{r^{\prime}}\left(z_{n}\right),\left|\operatorname{supp} \zeta_{n}\right| \leq k \gamma_{n}$ for any $n \in \mathbb{N}$, and there exists $\mathbb{A} \in \mathcal{U}$ such that $\left\|\lambda_{n}(a) \zeta_{n}\right\|>\left\|\lambda_{n}(a)\right\|-\varepsilon$ for any $n \in \mathbb{A}$.

Note that $y \in \widetilde{B}_{r^{\prime}}\left(z_{n}\right)$ if $l_{n}\left(z_{n}^{-1} y\right) \leq r^{\prime}$.

Let $\alpha_{n}=\min _{g \in G_{n}, g \neq e} l(g)$. It follows from $\cap_{n=1}^{\infty} G_{n}=\{e\}$ that $\lim _{n \rightarrow \infty} \alpha_{n}=\infty$. The following statement is folklore.

Lemma 4.2. Let $g \in G, z=q_{n}(g)$, and let $B_{R}(g) \subset G$ be the ball of radius $R$ centered at $g$. Then $q_{n}$ maps $B_{R}(g)$ isometrically onto $\widetilde{B}_{R}(z)$ for any $R<\alpha_{n} / 4$. 
Proof. Let $h, k \in B_{R}(g)$. Then $d(h, k)=l\left(h^{-1} k\right) \leq 2 R$. Note that for any $x \in G$, $l(x) \geq l\left(q_{n}(x)\right)$, and $l_{n}\left(q_{n}(x)\right)=\min _{y \in G_{n}, y \neq e} l(x y)$. Suppose that there exists $y \in G_{n}$ such that $l(x y)<l(x)$. Then, by the triangle inequality, $l(x y) \geq l(y)-l(x)$, hence $l(y)-l(x)<l(x)$, or, equivalently, $l(x)>l(y) / 2$. Taking $x=h^{-1} k$, we get $2 R \geq d(h, k)=$ $l(x)>l(y) / 2 \geq \alpha_{n} / 2$, hence $R \geq \alpha_{n} / 4$ - a contradiction. Thus, $d_{n}\left(q_{n}(h), q_{n}(k)\right)=$ $l_{n}\left(q_{n}(x)\right)=l(x)=d(h, k)$.

Let $g_{n} \in G$ satisfy $q_{n}\left(g_{n}\right)=z_{n}, R<\alpha_{n} / 4$, and let $u_{n}: l^{2}\left(\widetilde{B}_{R}\left(z_{n}\right)\right) \rightarrow l^{2}(G)$ be an isometry defined by

$$
u_{n}\left(\varphi_{n}\right)(h)=\left\{\begin{array}{cl}
\varphi_{n}\left(q_{n}(h)\right) & \text { if } h \in \widetilde{B}_{R}\left(g_{n}\right) ; \\
0 & \text { if } h \notin \widetilde{B}_{R}\left(g_{n}\right) .
\end{array}\right.
$$

If the supports of $\varphi_{n}$ and of $\lambda_{n}(g) \varphi_{n}$ lie in $\widetilde{B}_{R}\left(z_{n}\right)$ then $u_{n} \lambda_{n}(g) \varphi_{n}=\lambda(g) u_{n} \varphi_{n}$. Both $\zeta_{n}$ and $\lambda_{n}(x) \zeta_{n}$ have supports in the ball $\widetilde{B}_{r^{\prime}+r}\left(z_{n}\right)$, hence, for $r^{\prime}+r<\alpha_{n} / 4$ one has $u_{n} \lambda_{n}(x) \zeta_{n}=\lambda(x) u_{n} \zeta_{n}$, so $\left\|\lambda_{n}(x) \zeta_{n}\right\|=\left\|\lambda(x) \zeta_{n}^{\prime}\right\|$, where $\zeta_{n}^{\prime}=u_{n} \zeta_{n} \in l^{2}(G)$.

Thus,

$$
\left\|\pi_{\gamma}(a)\right\|^{2}<\left\|\lambda_{n}(a) \zeta_{n}\right\|^{2}+\varepsilon=\left\|\lambda(a) \zeta_{n}^{\prime}\right\|^{2}+\varepsilon
$$

for any $n \in \mathbb{A}$ when $r^{\prime}+r<\alpha_{n} / 4$.

For any $\varepsilon>0$, there exists $\mathbb{A}^{\prime} \in \mathcal{U}$ such that

$$
\left\|\lambda(a) \zeta_{n}^{\prime}\right\|^{2}<\|\lambda(a)\|^{2}+\varepsilon
$$

for any $n \in \mathbb{A}^{\prime}$.

Choose $\gamma$ such that $\lim _{\mathcal{U}} \frac{\gamma_{n}}{\alpha_{n}}=0$. Then, as $r^{\prime}=3 r k \gamma_{n}$, so there exists $\mathbb{A}^{\prime \prime} \in \mathcal{U}$ such that $r^{\prime}+r<\alpha_{n} / 4$ holds for any $n \in \mathbb{A}^{\prime \prime}$. As the set $\mathbb{A} \cap \mathbb{A}^{\prime} \cap \mathbb{A}^{\prime \prime}$ is not empty, it follows from (4.3) and (4.4) that

$$
\left\|\pi_{\gamma}(a)\right\|^{2}<\|\lambda(a)\|^{2}+2 \varepsilon
$$

holds for any $a \in \mathbb{C}[G]$ with supp $a \in B_{r}$. As $r$ is arbitrary, we conclude that $\left\|\pi_{\gamma}(a)\right\| \leq$ $\|\lambda(a)\|$ for any $a \in \mathbb{C}[G]$.

\section{Case of small growth}

Let $U_{n-1}: l^{2}\left(X_{n-1}\right) \rightarrow l^{2}\left(X_{n}\right)$ be the isometry defined by

$$
U_{n-1}\left(\xi_{n-1}\right)(x)=\frac{1}{\sqrt{\nu_{n} / \nu_{n-1}}} \xi_{n-1}\left(q_{n}(x)\right),
$$

where $q_{n}: G / G_{n} \rightarrow G / G_{n-1}$ is the quotient homomorphism and $\nu_{n}=\left|X_{n}\right|$. This allows to consider $H_{n-1}$ as a subspace of $H_{n}$. Note that $\lambda_{n-1}$ is a subrepresentation of $\lambda_{n}$. Set $\rho_{n}=\lambda_{n} \ominus \lambda_{n-1}$. This is a representation of $G$ on $l^{2}\left(X_{n}\right) \ominus l^{2}\left(X_{n-1}\right)$.

Theorem 5.1. Let $\gamma \prec \gamma^{\prime}$, where $\gamma_{n}^{\prime}=\frac{\nu_{n}}{\nu_{n-1}}$. Then $\left\|\pi_{\gamma}(a)\right\| \leq \lim \sup _{n \rightarrow \infty}\left\|\rho_{n}(a)\right\|$ for any $a \in \mathbb{C}[G]$.

Proof. We are going to construct a Hilbert space $L$ such that $H_{\gamma} \subset L \subset H_{\nu}=\widehat{H}$ and a representation $\rho$ of $G$ on $L$ such that $\rho=\left.\widehat{\lambda}\right|_{L}$ and $\|\rho(a)\|=\lim _{\sup } \rightarrow \infty\left\|\rho_{n}(a)\right\|$ for any $a \in \mathbb{C}[G]$. This would obviously imply that $\rho$ contains $\pi_{\gamma}$, hence the claim of the theorem.

In order to construct $L$ let us consider the set $\widetilde{L}$ of all the sequences $\left(\xi_{n}\right)_{n \in \mathbb{N}}$ such that $\xi_{n} \in l^{2}\left(X_{n}\right) \ominus l^{2}\left(X_{n-1}\right)$ and the norms $\left\|\xi_{n}\right\|$ are uniformly bounded, with the degenerate inner product as before, which becomes positive definite after taking quotient modulo 
sequences with $\lim _{\mathcal{U}}\left\|\xi_{n}\right\|^{2}=0$. Then $\widetilde{L} \subset \widetilde{H}$, and we define $L$ as the closure of $\widetilde{L}+\widetilde{H}_{0} / \widetilde{H}_{0}$ in $\widehat{H}$. Then $\rho(g)\left(\xi_{n}\right)=\left(\rho_{n}(g) \xi_{n}\right), g \in G$, defines the representation $\rho$ on $L$.

Let $\sigma_{k}, k \in \mathbb{N}$, be the sequence of irreducible representations of $G$ that appear as direct summands in $\lambda_{1}, \lambda_{2}, \ldots$. Let $\sigma_{k}$ be a subrepresentation of $\lambda_{n-1}$. As the multiplicity of $\sigma_{k}$ in $\lambda_{n-1}$ and in $\lambda_{n}$ is the same and equals its dimension, it is not contained in $\rho_{n}$. Thus, each $\sigma_{k}$ appears only in one of $\rho_{1}, \rho_{2}, \ldots$ Let $\mathcal{B}$ be the $C^{*}$-algebra generated by all $\oplus_{n \in \mathbb{N}} \rho_{n}(g), g \in G$, in $H^{\prime}=\oplus_{n \in \mathbb{N}} l^{2}\left(X_{n}\right) \ominus l^{2}\left(X_{n-1}\right)$. Then $\|\rho(a)\|$ equals the norm of $a$ in $\mathcal{B} / \mathcal{B} \cap \mathbb{K}\left(H^{\prime}\right)$ by Theorem 5.3 of [3]. Since each $\sigma_{k}$ appears only in one of $\rho_{1}, \rho_{2}, \ldots$, the latter norm equals $\lim \sup _{k \rightarrow \infty}\left\|\sigma_{k}(a)\right\|=\limsup _{n \rightarrow \infty}\left\|\rho_{n}(a)\right\|$.

To finish the argument, it remains to show that $H_{\gamma} \subset L$. Let $P_{n}: l^{2}\left(X_{n}\right) \rightarrow l^{2}\left(X_{n}\right) \ominus$ $l^{2}\left(X_{n-1}\right)$ be the orthogonal projection, $Q_{n}=1-P_{n}$. We claim that $\lim _{\mathcal{U}}\left\|\xi_{n}-P_{n} \xi_{n}\right\|=0$ for any $\xi \in H_{\gamma}$. Let $\xi \in \widetilde{H}_{\gamma}^{(k)}$ for some $k \in \mathbb{N}$.

Let $x \in X_{n-1}=G / G_{n-1}, \delta_{x}$ its characteristic delta-function, $\eta_{x}=U_{n-1} \delta_{x} \in l^{2}\left(X_{n}\right)$. Then $Q_{n} \xi_{n}=\sum_{x \in X_{n-1}}\left\langle\eta_{x}, \xi_{n}\right\rangle \eta_{x}$. Note that $\eta_{x}(y)=\left\{\begin{array}{cl}\frac{1}{\sqrt{\nu_{n} / \nu_{n-1}}} & \text { if } q_{n}(y)=x \\ 0 & \text { if } q_{n}(y) \neq x\end{array}\right.$

Note also that $\operatorname{supp} \eta_{x} \cap \operatorname{supp} \eta_{y}=\emptyset$ when $x \neq y$. Let $A_{x}=\operatorname{supp} \xi_{n} \cap \operatorname{supp} \eta_{x} \subset X_{n}$. Then $\operatorname{supp} \xi_{n}=\sqcup_{x \in X_{n-1}} A_{x}$, hence $\sum_{x \in X_{n-1}}\left|A_{x}\right| \leq k \gamma_{n}$.

$$
\begin{aligned}
\left\|Q_{n} \xi_{n}\right\|^{2} & =\sum_{x \in X_{n-1}}\left|\left\langle\eta_{x}, \xi_{n}\right\rangle\right|^{2} \leq \sum_{x \in X_{n-1}} \sum_{y \in A_{x}}\left|\eta_{x}(y)\right|^{2} \sum_{y \in A_{x}}\left|\xi_{n}(y)\right|^{2} \\
& \leq \sum_{x \in X_{n-1}}\left|A_{x}\right| \frac{1}{\nu_{n} / \nu_{n-1}}\left\|\xi_{n}\right\|^{2} \leq k \gamma_{n} \frac{\nu_{n-1}}{\nu_{n}}\left\|\xi_{n}\right\|^{2} .
\end{aligned}
$$

So, $\lim _{\mathcal{U}}\left\|Q_{n} \xi_{n}\right\|=0$, hence $\xi \in \widetilde{L}$.

\section{Case of intermediate Growth}

Here we consider the case of intermediate growth and show two different kinds of behaviour of representations $\pi_{\gamma}$ - for property $(\mathrm{T})$ groups and for free groups.

6.1. Case of property $(\tau)$ groups. Recall that the property $(\tau)$ is a generalization of the property $(\mathrm{T})$ of Kazhdan and means that the trivial representation is isolated among finitedimensional representations. For more details about property $(\tau)$ we refer to [4].

Theorem 6.1. Let $G$ be a finitely generated property $(\tau)$ group, and let $\gamma \prec \nu$. Then the trivial representation is not weakly contained in $\pi_{\gamma}$.

Proof. Let $S \subset G$ be a finite symmetric generating set, and let $x=\frac{1}{|S|} \sum_{g \in S} g \in \mathbb{C}[G]$. Suppose the contrary: for any $\varepsilon>0$ there exists $\xi^{(k)} \in \widetilde{H}_{\gamma}$ such that $\left\|\xi^{(k)}\right\|=1$ and $\left\|\pi_{\gamma}(x) \xi^{(k)}-\xi^{(k)}\right\|<\varepsilon$. Without loss of generality we may assume that $\xi^{(k)} \in \widetilde{H}_{\gamma}^{(k)}$, and that $\left\|\xi_{n}\right\|=1$ for each $n \in \mathbb{N}$, where $\xi=\left(\xi_{n}\right)_{n \in \mathbb{N}}$. A fixed $\varepsilon$ determines $k$ such that

$$
\left\|\pi_{\gamma}(x) \xi^{(k)}-\xi^{(k)}\right\|=\lim _{\mathcal{U}}\left\|\lambda_{n}(x) \xi_{n}^{(k)}-\xi_{n}^{(k)}\right\|<\varepsilon
$$

Then there exists $\mathbb{A} \in \mathcal{U}$ such that

$$
\left\|\lambda_{n}(x) \xi_{n}^{(k)}-\xi_{n}^{(k)}\right\|<\varepsilon
$$

for any $n \in \mathbb{A}$.

Note that each $\lambda_{n}$ contains exactly one copy of the trivial representation, with the representation space spanned by the single vector $\xi_{n}^{0}=\frac{1}{\sqrt{\left|X_{n}\right|}} \chi_{X_{n}}$. By property $(\tau)$, there 
exists $\delta>0$ (which does not depend on $n$ ) such that $\left\|\lambda_{n}(x) \eta_{n}-\eta_{n}\right\| \geq \delta\left\|\eta_{n}\right\|$ for any $\eta_{n} \in l^{2}\left(X_{n}\right)$ orthogonal to $\xi_{n}^{0}$. Let $\xi_{n}^{(k)}=\alpha \xi_{n}^{0}+\eta_{n}$, where $\eta_{n} \perp \xi_{n}^{0}$. Then

$$
\delta\left\|\eta_{n}\right\| \leq\left\|\lambda_{n}(x) \eta_{n}-\eta_{n}\right\|=\left\|\lambda_{n}(x) \xi_{n}^{(k)}-\xi_{n}^{(k)}\right\|<\varepsilon
$$

for any $n \in \mathbb{A}$, hence $\left\|\eta_{n}\right\|<\frac{\varepsilon}{\delta}$ when $n \in \mathbb{A}$. Then $|\alpha|=\sqrt{1-\left\|\eta_{n}\right\|^{2}}>\sqrt{1-\frac{\varepsilon^{2}}{\delta^{2}}}$, and

$$
\left\|\xi_{n}^{(k)}-\xi_{n}^{0}\right\|=\sqrt{(1-\alpha)^{2}+\left\|\eta_{n}\right\|^{2}}<\sqrt{2 \frac{\varepsilon}{\delta}}
$$

when $n \in \mathbb{A}$ and $\varepsilon \leq \delta$.

But $\left|\operatorname{supp} \xi_{n}^{(k)}\right| \leq k \gamma_{n}$ for any $n \in \mathbb{N}$, hence $\left|\left\langle\xi_{n}^{(k)}, \xi_{n}^{0}\right\rangle\right| \leq \sqrt{\frac{k \gamma_{n}}{\left|X_{n}\right|}}$. So, by assumption, $\lim _{n \rightarrow \infty}\left\langle\xi_{n}^{(k)}, \xi_{n}^{0}\right\rangle=0$, which contradicts (6.2) when $\varepsilon<\delta / 4$.

6.2. Case of free groups. Let $G=\mathbb{F}_{2}$ be the free group on two generators. Here we show that $H_{\gamma}$ may weakly contain the trivial representation for a certain sequence of finite index subgroups and for certain intermediate $\gamma$. We follow here [1].

Let $G_{0}=\mathbb{F}_{2}, G_{1}=\mathbb{F}_{2}^{(2)}, G_{n+1}=G_{n}^{(2)}$, be the iterated subgroups generated by the squares of all elements of the previous group. There is a nice description of $X_{n}=\mathbb{F}_{2} / G_{n}$ in [1] as vertices of Cayley graphs of $X_{n}$. The Cayley graph of $\mathbb{F}_{2} / G_{0}$ is the wedge of two circles with a single vertex, and the Cayley graphs $\operatorname{Cay}\left(X_{n}\right)$ of $\mathbb{F}_{2} / G_{n}=X_{n}$ are constructed from it inductively. Let $V_{n}$ and $E_{n}$ denote the set of vertices and edges of the Cayley graph of $X_{n}$, let $T_{n}$ be a maximal tree in $\operatorname{Cay}\left(X_{n}\right)$, and let $e_{1}, \ldots, e_{r_{n}}$ be the edges not in $T_{n}$. Then $V_{n+1}=V_{n} \times(\mathbb{Z} / 2)^{r_{n}}, E_{n+1}=E_{n} \times(\mathbb{Z} / 2)^{r_{n}}$. Let $e \in E_{n}, \alpha \in(\mathbb{Z} / 2)^{r_{n}}$. Let $e$ connect the vertices $v, w \in V_{n}$. If $e \in T_{n}$ then the edge $(e, \alpha) \in E_{n+1}$ connects $(v, \alpha)$ with $(w, \alpha)$. If $e=e_{i}, 1 \leq i \leq r_{n}$, then $(e, \alpha)$ connects $(v, \alpha)$ with $\left(w, \alpha+\bar{e}_{i}\right)$, where $\bar{e}_{i}=(0, \ldots, 0,1,0, \ldots, 0) \in(\mathbb{Z} / 2)^{r_{n}}$ has 1 as its $i$ 's component.

For a Cayley graph $\operatorname{Cay}\left(X_{n}\right)$ and for a subset $A$ of vertices of $\operatorname{Cay}\left(X_{n}\right)$ we denote by $|\partial A|$ the number of edges in $C a y\left(X_{n}\right)$ such that they connect a point from $A$ with a point from $X_{n} \backslash A$.

Lemma 6.2. There exists a sequence $A_{n} \subset X_{n}$ such that

$$
\begin{aligned}
& \lim _{n \rightarrow \infty}\left|A_{n}\right|=\infty ; \\
& \lim _{n \rightarrow \infty} \frac{\left|A_{n}\right|}{\left|X_{n}\right|}=0 ; \\
& \lim _{n \rightarrow \infty} \frac{\left|\partial A_{n}\right|}{\left|A_{n}\right|}=0 .
\end{aligned}
$$

Proof. Let $1<k_{n}<r_{n}$. Set

$$
A_{n+1}=\left\{(v, \alpha): v \in V_{n}, \alpha \in(\mathbb{Z} / 2)^{r_{n}}, \alpha_{i}=0 \text { for } k_{n}<i \leq r_{n}\right\} .
$$

Then $\left|A_{n+1}\right|=\left|X_{n}\right| 2^{k_{n}}$, and (6.3) holds for any $k_{n} \geq 1$. Note that (6.4) means that

$$
\lim _{n \rightarrow \infty} \frac{\left|A_{n+1}\right|}{\left|X_{n+1}\right|}=\lim _{n \rightarrow \infty} 2^{k_{n}-r_{n}}=0,
$$

which is equivalent to $\lim _{n \rightarrow \infty}\left(r_{n}-k_{n}\right)=\infty$.

Let us evaluate $\left|\partial A_{n+1}\right|$. If $e \in T_{n}$ then both ends of $(e, \alpha)$ are either in $A_{n+1}$ or in $V_{n+1} \backslash A_{n+1}$, so let $e=e_{i}$ be one of $e_{1}, \ldots, e_{r_{n}}$. If $(e, \alpha) \in \partial A_{n+1}$ then $i>k_{n}$, so $\left|\partial A_{n+1}\right|=2\left(r_{n}-k_{n}\right) 2^{k_{n}}$. 
Thus, $\frac{\left|\partial A_{n+1}\right|}{\left|A_{n+1}\right|}=\frac{2\left(r_{n}-k_{n}\right)}{\left|X_{n}\right|}$. As both $\left(r_{n}\right)_{n \in \mathbb{N}}$ and $\left(\left|X_{n}\right|\right)_{n \in \mathbb{N}}$ grow faster than an iterated exponential, so one can easily find a sequence $\left(k_{n}\right)_{n \in \mathbb{N}}$ such that

- $\lim _{n \rightarrow \infty}\left(r_{n}-k_{n}\right)=\infty$;

- $\lim _{n \rightarrow \infty} \frac{\left(r_{n}-k_{n}\right)}{\left|X_{n}\right|}=0$

(the latter implies (6.5) ).

Theorem 6.3. There exists $\gamma$ with $\lim _{n \rightarrow \infty} \gamma_{n}=\infty$ and $\lim _{n \rightarrow \infty} \frac{\gamma_{n}}{d_{n}}=0$ such that $\pi_{\gamma}$ weakly contains the trivial representation.

Proof. Let $A_{n} \subset X_{n}$ be as in Lemma 6.2. Set $\gamma_{n}=\left|A_{n}\right|$, and $\xi_{n}=\frac{1}{\sqrt{\gamma_{n}}} \chi_{A_{n}}$. Then $\xi=\left(\xi_{n}\right)_{n \in \mathbb{N}} \in \widetilde{H}_{\gamma}$. If $g \in S \subset G$ is one of the generators then

$$
\begin{aligned}
\left\|\lambda_{n}(g) \xi_{n}-\xi_{n}\right\|^{2} & \leq \frac{1}{\gamma_{n}}\left(\left|g\left(A_{n}\right) \backslash A_{n}\right|+\left|A_{n} \backslash g\left(A_{n}\right)\right|\right) \\
& =\frac{1}{\gamma_{n}}\left(\left|g\left(A_{n}\right) \backslash A_{n}\right|+\left|g^{-1}\left(A_{n}\right) \backslash A_{n}\right|\right) \leq \frac{2\left|\partial A_{n}\right|}{\gamma_{n}}
\end{aligned}
$$

hence $\xi$ is almost invariant for $\pi_{\gamma}$ by Lemma 6.2.

\section{REFERENCES}

[1] G. Arzhantseva, E. Guentner. Coarse non-amenability and covers with small eigenvalues. Math. Ann. 354 (2012), 863-870.

[2] N. P. Brown, E. P. Guentner. New $C^{*}$-completions of discrete groups and related spaces. Bull. Lond. Math. Soc. 45 (2013), 1181-1193.

[3] J. W. Calkin. Two-sided ideals and congruences in the ring of bounded operators in Hilbert space. Ann. Math. 42 (1941), 839-873.

[4] A. Lubotzky. Discrete groups, expanding graphs and invariant measures. Progress in Math. 125, Birkhäuser Verlag, Basel (1994).

[5] S. Wassermann. On tensor products of certain group $C^{*}$-algebras. J. Funct. Anal. 23 (1976), 239-254.

[6] M. Wiersma. Constructions of exotic group $C^{*}$-algebras. Illinois J. Math. 60 (2016), 655-667.

Moscow State University, Leninskie Gory 1, Moscow, 119991, Russia

E-mail address: manuilov@mech.math.msu.su 